\title{
Prevalência e posição de caninos superiores impactados e sua relação com reabsorção radicular
}

\author{
Prevalence and position of impacted maxillary canines and their relation \\ with root resorption
}

\author{
Eduardo Peterini Alves* \\ Anelise Fernandes Montagner ${ }^{* *}$ \\ Simone Pippi Antoniazzi $i^{* * *}$ \\ Luiz Felipe Durand de Oliveira**
}

\section{Resumo}

Objetivo: este estudo teve como objetivo avaliar a posição e a prevalência de caninos maxilares inclusos superiores bem como a presença de reabsorção radicular em dentes adjacentes. Materiais e método: foram examinadas 372 radiografias panorâmicas, de pacientes entre 13 e 50 anos de idade, realizadas, entre os anos de 2008 e 2013, em duas instituições de ensino. As radiografias foram examinadas por um único avaliador, com o auxílio de negatoscópio e lupa com diâmetro de 90 mm e aumento de 2,5 vezes. Os dados foram submetidos à análise estatística pelo teste Qui-Quadrado ( $\alpha=5 \%$ ). Resultado: foram encontradas um total de 13 radiografias com presença de caninos superiores inclusos, portanto, uma prevalência de $3,5 \%$. A idade foi um fator que, estatisticamente, influenciou na presença de caninos inclusos $(p=0,040)$, sendo mais prevalente em pacientes com idade de 13 a 30 anos do que em pacientes de 31 a 50 anos. A retenção de caninos foi mais recorrente no gênero feminino, porém, sem significância estatística ( $p$ $=0,163)$. A reabsorção do dente adjacente ocorreu em 15,4\% dos casos. Conclusão: a prevalência de caninos inclusos foi dependente da idade, a qual influenciou a presença de caninos inclusos, e a posição mésio-angulada foi predominante em todos os casos.

Palavras-chave: Dente canino. Dente não erupcionado. Reabsorção da raiz.

\section{Introdução}

O processo de erupção dental e o movimento do dente à posição funcional final na cavidade oral depende de uma série de eventos ${ }^{1}$. Por razões multifatoriais, quando chegada a época de erupção, o dente pode ficar recluso parcial ou totalmente no interior do osso, com manutenção ou não da integridade do saco pericoronário, caracterizando-se como dente retido/incluso (dente intra-ósseo ou submucoso) ou impactado (dente obstruído por algum objeto) ${ }^{2}$. Dentre os dentes mais acometidos por essa anomalia, encontram-se os terceiros molares e os caninos permanentes ${ }^{3}$. A abordagem do estudo dos caninos inclusos tem grande relevância na Ortodontia, considerada a grande preocupação em tracionar ortodonticamente o canino retido, pela importância estratégica desse dente no arco dentário, devido sua função nas relações oclusais e estéticas. O diagnóstico e a intervenção precoces, quanto a essa anomalia, podem reduzir, ou evitar, possíveis complicações tardias ${ }^{4}$.

Como fatores gerais, a literatura aponta como principais causas de retenção de caninos os fatores hereditários, a raça, os distúrbios endócrinos e as síndromes com má-formação craniofaciais. Como causas locais, os seguintes fatos são relatados: tra-

Estudante de especialização em Ortodontia, Faculdade de Odontologia, Centro Universitário Franciscano (Unifra), Santa Maria-RS, Brasil.

Estudante de doutorado em Odontologia, Faculdade de Odontologia, Universidade Federal de Pelotas, Pelotas-RS, Brasil.

* Professor do curso de especialização em Ortodontia, Faculdade de Odontologia, Centro Universitário Franciscano (Unifra), Santa Maria-RS, Brasil. 
jeto de irrupção longo e tortuoso, pois é é um dos últimos dentes a irromper na cavidade bucal; falta de espaço no arco dentário; distúrbios na sequência de irrupção dos dentes permanentes; trauma dos dentes decíduos; agenesia dos incisivos laterais permanentes; má-posição do germe dentário; dilaceração radicular e a anquilose dos caninos permanentes; retenção prolongada ou perda prematura do canino decíduo predecessor e presença de cistos; tumores ou supranumerários na região - servindo como obstáculo - e a fissura alveolar ${ }^{5,6}$.

Estudos têm revelado que os caninos apresentam alta frequência de impacção, ou de deslocamento, sendo a ocorrência da maioria dos casos no gênero feminino $0^{7}$, e em descendentes europeus ${ }^{8}$. A prevalência de retenção ocorre nas seguintes condições: de $0,9 \%$ a $2,5 \%$ com maior frequência unilateral; de $75 \%$ a $95 \%$ dos casos no gênero feminino duas a três vezes mais que no gênero masculino; de 60 a $80 \%$ dos casos estão localizados por palatino 9 . A impaç̧ão de caninos é 10 vezes maior na maxila do que na mandíbula ${ }^{10,11}$, sendo o lado esquerdo da arcada o mais afetado ${ }^{12}$. Os caninos podem estar em posições variadas de retenção, como vertical, horizontal e, muito raramente, invertidos ${ }^{13}$. A impacção usualmente envolve um único canino permanente, contudo 8\% dos casos são bilaterais ${ }^{7}$.

Dentre os diversos problemas causados por tal anomalia, a reclusão dos caninos pode influenciar na reabsorção dos dentes adjacentes. Essa reabsorção tem sido relatada nos estudos como resultado do efeito físico do contato entre o canino impactado e o dente adjacente, devido à pressão ativa, durante a erupção ${ }^{14}$. Estudos mostraram que aproximadamente $12 \%$ dos incisivos adjacentes são reabsorvidos pelos caninos ectópicos, sendo a prevalência quatro vezes maior em meninas do que em meninos ${ }^{7,15}$. Porém, essa reabsorção, as vezes, também pode ocorrer quando o canino erupciona normalmente ${ }^{4}$.

As tomadas radiográficas panorâmicas são fundamentais na localização de caninos retidos, os quais quando localizados por palatina apresentam imagem maior e mais nítida, e, em aproximadamente $90 \%$ dos casos, consegue-se localizar os caninos retidos apenas com o uso desse tipo de imagem ${ }^{16}$. A posição que tal dente se encontra pode proporcionar futuras complicações e determinar o tipo de tratamento aplicado para tracioná-lo, além de influenciar na reabsorção radicular ${ }^{4}$

Tendo em vista todos os fatores supracitados, o objetivo geral deste estudo foi avaliar, através de radiografias panorâmicas, a posição e a prevalência de caninos maxilares inclusos e a presença de reabsorção radicular visível nos dentes adjacentes; bem como, avaliar a interferência do gênero, da raça e da faixa etária.

\section{Materiais e método}

Este trabalho foi avaliado e aprovado pelo Comitê de Ética em Pesquisa do Centro Universitário Franciscano (parecer 344.432) com certificado de apresentação para apreciação ética (CAAE) de $n$. 15875013.1.0000.5306, sendo conduzido em agosto de 2013.

O cálculo amostral foi realizado no programa Sigmastat $^{\circledR}$ (Versão 3.01, Systat Software Inc., San Jose, CA, USA). Considerando uma diferença entre as médias de prevalência de caninos superiores retidos de 1,7 e um desvio padrão médio de 0,9 e também tais parâmetros, com poder de $80 \%$ e nível de significância de $5 \%$, obteve-se um n amostral de 372 radiografias.

Neste estudo retrospectivo foram avaliadas 372 radiografias panorâmicas da documentação ortodôntica inicial, de pacientes com idade entre 13 e 50 anos, os quais foram tratados entre os anos de 2008 e 2013. As radiografias foram coletadas em duas instituições de ensino de Santa Maria (curso de Pós-Graduação em Ortodontia da Uningá e do Centro Universitário Franciscano). Os pacientes foram divididos categoricamente, de acordo com a faixa etária, em dois grupos: dos 13 aos 30 anos e dos 31 aos 50 anos. Foram analisadas as radiografias panorâmicas iniciais de todos os pacientes que se encaixaram nos critérios de inclusão supracitados, independente do estágio em que se encontrava o tratamento.

A amostra foi considerada de conveniência, uma vez que um pesquisador selecionou as 372 radiografias mais acessíveis de todo o universo de radiografias. Foram escolhidas apenas radiografias nas quais a região, ou as regiões em que se encontram os dentes inclusos puderam ser visualizadas de forma clara. Foram excluídas as radiografias que não se apresentaram em adequada situação, ou que não possibilitaram a avaliação dos critérios propostos (inclusão do dente canino superior, posição e reabsorção radicular).

As pastas do Centro Universitário Franciscano foram todas avaliadas, o que não foi possível realizar com as pastas da Uningá, devido à grande quantidade de pastas, assim, foi necessária uma seleção aleatória dessas. Todas as radiografias panorâmicas foram avaliadas por um único examinador treinado e experiente, em ambiente com adequada luminosidade, com auxílio de um negatoscópio com lupa de $90 \mathrm{~mm}$ de diâmetro e aumento de 2,5 vezes para a visualização dos dentes retidos.

Os desfechos primários considerados foram a posição e a prevalência do dente canino superior incluso e a presença de reabsorção radicular nos dentes adjacentes, na amostra estudada. Considerou-se como dente incluso aquele que apresentou as seguintes características: 
a) permanência prolongada de dentes decíduos;

b) perda prematura de dentes decíduos, alterando a posição dos permanentes;

c) presença do dente intra-ósseo com alteração da direção do seu longo eixo.

Em caso de dúvidas na análise radiográfica dos caninos, fotos intrabucais contidas na documentação ortodôntica inicial dos pacientes foram analisadas para confirmar a inclusão desse.

A posição dos dentes caninos inclusos e a presença de reabsorção dos dentes maxilares adjacentes foram documentadas bilateralmente. A posição do canino foi definida seguindo uma adaptação da Classificação de Winter ${ }^{17}$ (Figura 1):

a) vertical - quando estiverem paralelos em relação ao longo eixo do incisivo lateral;

b) mesioangular - quando o longo eixo do dente estiver em posição medial em relação ao longo eixo do incisivo lateral;

c) distoangular - quando o longo eixo do dente estiver em posição distal em relação ao longo eixo do incisivo lateral;

d) horizontal - quando o longo eixo do dente estiver perpendicular ao longo eixo do incisivo lateral.

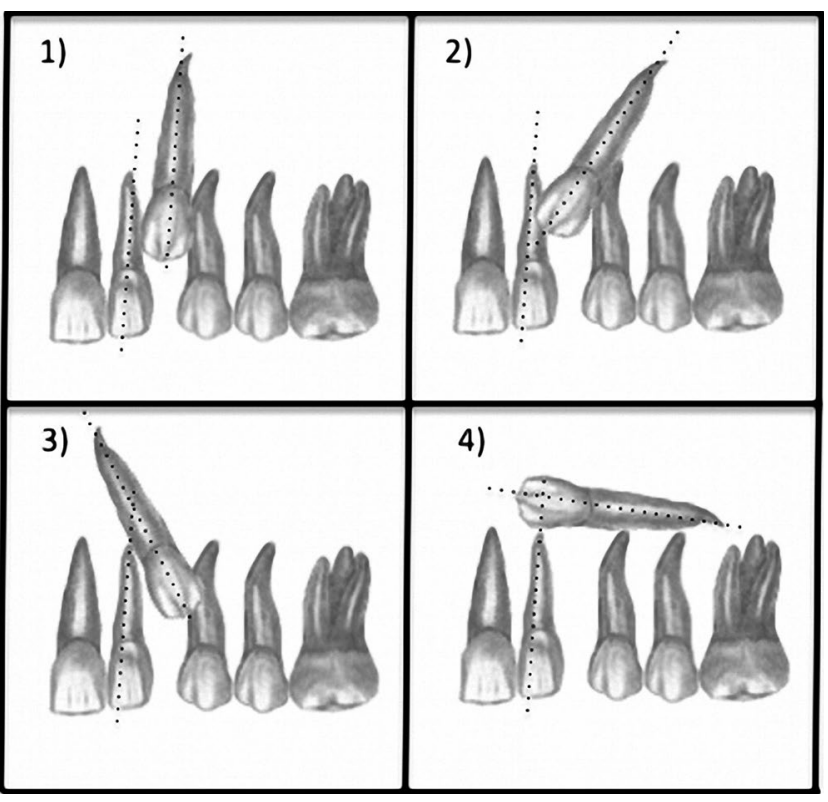

Figura 1 - Desenho esquemático dos caninos superiores retidos adaptado da Classificação de Winter para terceiros molares retidos.

A reabsorção radicular foi codificada em quatro categorias baseadas no estudo de Ericson ${ }^{4}$ :

a) nenhuma reabsorção: superfícies da raiz intacta. A camada de cemento pode ser perdida;

b) reabsorção leve: até a metade da espessura de dentina;

c) reabsorção moderada: reabsorção perto da polpa ou mais, a linha da polpa sendo quebrada;

d) reabsorção severa: a polpa exposta pela reabsorção.
E os desfechos secundários considerados foram gênero, raça e faixa etária.

Todos os dados foram anotados em ficha especialmente elaborada para esse fim e, posterioremente, tabulados em planilhas.

\section{Análise estatística}

A análise estatística foi conduzida no programa estatístico SPSS (Statistical Package for Social Sciences, versão 18.0, Chicago, IL, USA). A homogeneidade da amostra foi verificada através do teste de Levene. A estatística descritiva foi utilizada para determinar a prevalência e a respectiva influência de cada fator sobre essa foi verificada com a aplicação do teste estatístico Qui-Quadrado, considerando nível de significância de $5 \%$ e poder de $80 \%$.

\section{Resultados}

Foram analisadas 372 radiografias panorâmicas, dessas foram encontradas 13 radiografias com presença de caninos superiores retidos, totalizando uma prevalência de 3,5\% na população estudada.

A idade foi um fator que, estatisticamente, influenciou a presença de caninos retidos $(p=0,040)$, sendo mais prevalente em pacientes com idade de 13 a 30 anos do que em pacientes com idade mais avançada, de 31 a 50 anos (Tabela 1). Entretanto, não houve diferenças estatisticamente significantes para os outros fatores avaliados nesse estudo, como raça $(p=0,932)$, gênero $(p=0,163)$ e local $(p=0,471)$. $\mathrm{O}$ gênero foi um fator que estatisticamente não influenciou na presença de caninos retidos, visto que teve-se 10 em mulheres $(4,5 \%)$ e 3 em homens $(2,1 \%)$.

Das 13 radiografias panorâmicas com presença de caninos inclusos, apenas uma radiografia apresentou reclusão bilateral, as demais eram unilaterais, além disso, estatisticamente, não houve um lado que se possa considerar ter sido mais acometido do que o outro por essa anomalia.

A posição do dente teve influência estatisticamente significante $(p<0,05)$, em que se tem a posição mésio-angulada como a mais frequente, incidindo em $100 \%$ dos casos.

A presença de reabsorção radicular visível nos dentes adjacentes não foi estatisticamente significante, ocorrendo em apenas dois casos, dos treze estudados $(15,4 \%)$. 
Tabela 1 - Características da população estudada e a prevalência de caninos retidos em cada fator avaliado

\begin{tabular}{l|l|c|c|c}
\hline Características & \multicolumn{1}{|c|}{ Subdivisão } & Presença de canino retidon [\%] & Ausência de canino retido $\mathrm{n}[\%]$ & $\mathrm{n}[\%]$ \\
\hline \multirow{2}{*}{ Idade } & 13 a 30 anos & $13[4,5 \%]$ & $279[95,5 \%]$ & $292[100 \%]$ \\
& 31 a 50 anos & $0[0 \%]$ & $80[100 \%]$ & $80[100 \%]$ \\
\hline \multirow{2}{*}{ Gênero } & Masculino & $3[2,1 \%]$ & $146[97,9 \%]$ & $149[100 \%]$ \\
& Feminino & $10[4,5 \%]$ & $213[95,5 \%]$ & $223[100 \%]$ \\
\hline \multirow{2}{*}{ Raça } & Leucoderma & $12[3,4 \%]$ & $338[96,6 \%]$ & $350[100 \%]$ \\
& Melanoderma & $1[4,8 \%]$ & $20[95,2 \%]$ & $21[100 \%]$ \\
& Chantoderma & $0[0 \%]$ & $1[100 \%]$ & $1100 \%]$ \\
\hline \multirow{2}{*}{ Local } & Unifra & $4[4,1 \%]$ & $95[95,9 \%]$ & $99[100 \%]$ \\
& Uningá & $9[3,3 \%]$ & $264[96,7 \%]$ & $273[100 \%]$ \\
\hline
\end{tabular}

\section{Discussão}

No presente estudo, foi encontrada prevalência de $3,5 \%$ de caninos inclusos na população estudada. Mostrando-se uma prevalência maior do que em alguns estudos encontrados, que apontaram dados de prevalências que variaram de $2,5 \%$ a $3 \% \%^{7,9,12,18}$. Devido às características da amostra por conveniência, não se deve excluir a presença de viés por amostragem.

Neste estudo, dos treze casos encontrados de caninos inclusos na população estudada, dez ocorreram em mulheres e apenas três em homens. Entretanto, mesmo que a maioria dos casos de caninos inclusos ocorram em mulheres, neste estudo, o gênero não foi um fator estatisticamente significante para a presença de caninos inclusos, muito provavelmente devido à grande maioria da população ser feminina. A maioria dos estudos presentes na literatura acerca desse tema demonstra que o gênero feminino é o mais acometido por essa anomalia ${ }^{10-12}$, como citado, por exemplo, no estudo de Garib et al. ${ }^{9}$ em que de $75 \%$ a $95 \%$ dos casos ocorreram com o gênero feminino, duas a três vezes mais do que no gênero masculino. $\mathrm{O}$ mesmo resultado aconteceu no presente estudo, no qual 76,9\% dos casos foram em mulheres. No entanto, há que se destacar que um estudo aleatório israelita apontou números equivalentes entre homens e mulheres ${ }^{19}$.

O achado de que a maioria dos casos são unilaterais ${ }^{11,12}$ também foi comprovado por este estudo, ocorrendo em 12 dos 13 casos encontrados, gerando um dado de apenas $7,7 \%$ de retenção bilateral de caninos na amostra avaliada. Resultado similar ao encontrado no estudo de Turner e $\mathrm{Bedi}^{7}$, no qual a retenção, na maioria dos casos, envolveu um único canino permanente, contudo, $8 \%$ dos casos são bilaterais. Com relação ao lado mais acometido, nesse trabalho não houve diferença estatística, ocorrendo presença de canino incluso unilateral em $46,2 \%$ dos casos, tanto no lado direito, quanto no esquerdo. Esses achados divergiram da literatura, em que um estudo apontou o lado esquerdo ${ }^{12}$ e outro o lado direito como o mais acometido por essa anomalia ${ }^{20}$.

Dos casos registrados de caninos inclusos, $100 \%$ apresentaram a posição mésio-angulada, ou seja, quando o longo eixo do canino incluso encontrava-se em posição medial em relação ao longo eixo do incisivo lateral, corroborando os estudos em que se avaliou a angulação dos caninos inclusos, por meio do ângulo formado pela linha que passava pelo longo eixo do canino e por aquela que passava entre os incisivos centrais. Com isso, evidencia-se a prevalência da posição mésio-angulada do canino incluso em uma amostra de setenta casos de impacção, sendo 55 casos unilaterais e 15 bilaterais, todos apresentando caninos mésio-angulados ${ }^{20}$. Outros estudos mostraram variações na angulação do canino incluso de $0^{\circ}$ a $30^{\circ}$ e $15^{\circ}$ a $45^{\circ}$, mas sempre prevalecendo a posição mésio-angulada ${ }^{21-22}$.

Com relação à reabsorção radicular, neste estudo $15,4 \%$ dos casos de caninos retidos apresentaram determinado grau de reabsorção, revelando-se presente exclusivamente no gênero feminino e na faixa etária dos 13 aos 30 anos de idade. Assim, demonstraram-se resultados semelhantes aos da literatura, em que uma amostra de 11 pacientes com impacção de caninos, a reabsorção radicular foi encontrada quase que exclusivamente em mulheres, com idades variando entre 11 e $23 \operatorname{anos}^{23}$. Outros estudos, realizados com amostras maiores também registraram maior prevalência no gênero feminino $0^{9,15,24}$ e acometendo uma faixa de idade que varia dos 11 aos $30 \operatorname{anos}^{24,25}$.

Convergindo com os dados discutidos anteriormente, a idade foi um fator que estatisticamente influenciou na presença de caninos retidos $(p=0,040)$. Com $100 \%$ dos casos de caninos retidos acometendo o grupo de faixa etária dos 13 aos 30 anos,concordando com a maioria dos achados na literatura ${ }^{13,15,23,24}$. Uma hipótese para essa prevalência presente no grupo de faixa etária dos 13 aos 30 anos é que, talvez, o canino incluso possa ter erupcionado tardiamente com o avançar da idade, ou, que os indivíduos tenham procurado tratamento, como a exodontia do canino incluso.

\section{Conclusão}

Dentro das limitações deste estudo, diante dos resultados obtidos e da metodologia empregada, concluímos que, na amostra estudada, a prevalência de caninos superiores retidos foi de $3,5 \%$, sendo todos os casos na mesma posição e com baixa incidência de reabsorção do incisivo lateral. 


\section{Abstract}

Objective: this study aimed to evaluate the location and prevalence of maxillary canines included as well as the presence of root reabsorption on the adjacent teeth. Materials and method: 372 panoramic radiographs were examined from patients ranged from 13 up to 50 years old, performed among 2008 to 2013 in two dental schools. The radiographs were analysed by a single examiner, with the assistance of a magnifying and viewing box with a diameter of $90 \mathrm{~mm}$ and 2.5 times magnification. The data were statistically analysed by chi-square test $(\alpha=5 \%)$. Results: It was found a total of 13 radiographs with the presence of upper canines included resulting in a prevalence of $3.5 \%$. Age was a factor that statistically influenced the presence of canines included $(p=0.040)$, being more prevalent in patients of 13-30 years than in patients of 31-50 years old. The retention of canines was more prevalent in females, but without statistical significance $(p=0.163)$. The reabsorption of adjacent teeth occurred in $15.4 \%$ of cases. Conclusion: The prevalence of canine included was dependent of the age, which influenced the presence of canines included, and the mesiobuccal-angled position was predominant in all cases.

Keywords: Canine tooth. Tooth not erupted. Root reabsorption.

\section{Referências}

1. Kardos TB. The mechanism of tooth eruption. Br Dent $\mathrm{L}$ 1996; 181:91-5.

2. Marzola C. Retenção dental. 2. ed. São Paulo: Ed. Pancast 1995.

3. Mittal R, Rai D, Patil A, Garg A. An easy method of attachment to an impacted canine. Progress Orthod 2013, 3:11-14.

4. Ericson S, Kurol J. Resorption of incisors after ectopic eruption of maxillary canines. A CT study. Angle Orthod 2000; 70(6):415-423.

5. Msagati F, Simon ENM, Owibingire S. Pattern of occurrence and treatment of impacted teeth at the Muhimbili National Hospital. BMC Oral Health 2013, 13:37.

6. Peck S, Peck L, Kataja M. The palatally displaced canine as a dental anomaly of genetic origin. Angle Orthod 1994; 64(4):249-256

7. Turner JP, Bedi R. Combined orthodontic and restorative management of a case of bilateral ectopic canines and resorbed central incisors. Br Dent J 1996; 180(2):67-72.

8. Jacobs SG. The unerupted maxillary canine. Further observations on aetiology, radiographic localization, prevention/ interception, and when to suspect impaction. Aust Dent 1996; 41(5):310-316.

9. Garib DG, Henriques JFC, Freitas MR, Janson GRP. Caninos superiores retidos: Preceitos clínicos e radiográficos. Rev Dental Press Ortodon Ortop 1999; 4(4):14-20.

10. Aizenbud D. Bilateral maxillary impacted canines: a challenge for the lingual orthodontic technique. Lingual News 2003; 11:p5.

11. Maahs M, Berthold T. Etiologia, diagnóstico e tratamento de caninos superiores permanentes impactados. Rev Cienc Med Biol 2004; 3(1):130-138.

12. Tanaka O, Daniel RF, Vieira SW. O dilema dos caninos superiores impactados. Rev Ortod Gaúcha 2000; 4(2):122-128.
13. Katiyar R, Tandon P, Singh GP, Agrawal A, Chaturvedi TP. Management of impacted all canines with surgical exposure and alignment by orthodontic treatment. Contemp Clin Dent 2013; 4(3):371-373.

14. Ericson S, Krister B, Falahat B. Does the Canine Dental Follicle Cause Resorption of Permanent Incisor Roots? A Computed Tomographic Study of Erupting Maxillary Canines. Angle Orthod 2002; 72(2):95-104.

15. Ericson S, Kurol J. Incisor resorption caused by maxillary cuspids. A radiographic study. Angle Orthod 1987; 57:332-346.

16. Tormena-Júnior R, Filho MV, Ramalho AS, Wassall T, Valdrighi HC. Caninos superiores retidos: uma reabilitação estética e funcional. J Bras Ortodon Ortop Facial 2004; 9 (49):77-86.

17. Stivaros N1, Mandall NA. Radiographic factors affecting the management of impacted upper permanent canines. J Orthod. 2000; 27(2):169-173.

18. Al-Nimri K, Gharaibeh T. Space conditions and dental and occlusal features in patients with palatally impacted maxillary canines: an aetiological study. Eur J Orthod 2005; 27(5):461-465

19. Becker A. Tratamento ortodôntico de dentes impactados. In: Becker A. Caninos impactados por palatal. $4^{\mathrm{a}}$ ed. São Paulo: Santos 2004; 85-103.

20. Martins PP, Gurgel JA, Sant'Ana E, Júnior OF, Henriques JFC. Avaliação radiográfica da localização de caninos superiores não irrompidos. Rev Dent Press Ortodon Ortoped Facial 2005; 10(4):106-114.

21. Power SM, Orth M, Short MBE, Orth M. An investigation into the response of palatally displaced canines to the removal of deciduous canines and an assessment of factors contributing to favourable eruption. Br J Orthod 1993; 20(3):215-223.

22. Stewart JA, Heo G, Glover KE, Williamson PC, Lam Ewn, Major PW. Factors that relate to treatment duration for pacients with palatally impacted maxillary canines. Am J Orthod Dentofacial Orthop 2001; 119(3):216-225.

23. Sasakura H, Yoshida T, Murayama S, Hanada K, Nakajima $\mathrm{T}$. Root resorption of upper permanent incisor caused by impacted canine. Int J Oral Surg 1984; 13(4):299-306.

24. Howard RD. The displaced maxillary canine: positional varations associated with incisor resorption. Dent Pract 1972; 22:279-287.

25. Motamedi MH, Tabatabaie FA, Navi F, Shafeie HA, Fard BK, Hayati Z. Assessment of radiographic factors affecting surgical exposure and orthodontic alignment of impacted canines of the palate: a 15-year retrospective study. Oral Surg Oral Med Oral Pathol Oral Radiol Endod. 2009;107(6):772-775.

\section{Endereço para correspondência:}

Eduardo Peterini Alves

Rua Marechal Floriano Peixoto, 1494/701

CEP 97015-372 Santa Maria-RS

Fone: +55 55 9138-8820

E-mail: eduardopeterinialves@hotmail.com

Recebido: 12/01/2014. Aceito: 08/08/2014. 